\title{
Protein Kinase B
}

National Cancer Institute

\section{Source}

National Cancer Institute. Protein Kinase B. NCI Thesaurus. Code C41625.

Expressed in diverse tissues, Protein Kinase B (AKT/RAC Family) is a group (Alpha, Beta and Gamma) of cytoplasmic serine/threonine enzymes that covalently transfer the terminal, gamma phosphate group from ATP to a variety of substrate proteins and regulate cell signaling responses to insulin, PDGF, and IGF1 (through PI3K) involved in cell survival, cell proliferation, differentiation, apoptosis, glycogen synthesis, and glucose uptake. 